\title{
Lidil
}

Revue de linguistique et de didactique des langues

$38 \mid 2008$

Langue du droit

\section{Raisonnement linguistique chez les juges anglais : le cas de l'interprétation des testaments}

Ross Charnock

\section{OpenEdition}

Journals

Édition électronique

URL : http://journals.openedition.org/lidil/2781

DOI : 10.4000/lidil.2781

ISSN : $1960-6052$

Éditeur

UGA Éditions/Université Grenoble Alpes

Édition imprimée

Date de publication : 1 décembre 2008

Pagination : 109-124

ISBN : $978-2-84310-130-4$

ISSN : 1146-6480

\section{Référence électronique}

Ross Charnock, «Raisonnement linguistique chez les juges anglais : le cas de l'interprétation des testaments », Lidil [En ligne], 38 | 2008, mis en ligne le 01 juin 2010, consulté le 19 avril 2019. URL : http://journals.openedition.org/lidil/2781; DOI : 10.4000/lidil.2781

Ce document a été généré automatiquement le 19 avril 2019

(C) Lidil 


\title{
Raisonnement linguistique chez les juges anglais : le cas de l'interprétation des testaments
}

\author{
Ross Charnock
}

La fonction principale du juge est de décider de la bonne interprétation des textes. Pourtant, dans la 'common law' du moins, afin de ne pas juger au cas par cas, les juges évitent l'interprétation pragmatique. Selon la "règle littérale ", l'intention doit toujours s'appréhender à travers les mots utilisés. Cette règle traditionnelle pose naturellement des problèmes. Dans la pratique, en l'absence de critères contextuels, il s'avère souvent difficile de déterminer le sens des expressions référentielles. En effet, si tout énoncé est associé quasiment par définition à un contexte, ce contexte doit toujours jouer un rôle dans la compréhension.

1 Concernant l'interprétation des lois écrites (statutes), l'objectif du juge est de donner effet à l'intention du législateur, mais il semble souvent difficile d'attribuer une intention commune à tous les membres du Parlement. D'une part, ils ne comprennent pas tous le texte de la même façon. D'autre part, certains n'ont pas envisagé les problèmes juridiques qui se posent, et n'ont donc pas d'avis sur la question. Il est donc normal de tenir compte de ce que le législateur a dit, plutôt que de faire des conjectures concernant ce qu'il aurait peut-être décidé s'il avait imaginé les circonstances particulières. C'est pourquoi Lord Reid précise, dans Black-Clawson $v$ Papierwerke 1975,qu'il faut rechercher non pas l'intention du législateur, ce qu'il a voulu dire, mais le « vrai sens de ce qu'il a dit ».

2 S'agissant de l'interprétation des testaments, l'objectif fondamental est toujours de respecter l'intention du testateur en se fondant sur les mots utilisés. Pourtant, depuis le jugement de James LJ dans l'affaire Boyes $v$ Cook 1880, il est admis que dans ce domaine le juge a la possibilité de se placer métaphoriquement dans le fauteuil du testateur afin de tenir compte de toutes les circonstances. Néanmoins, en règle générale, il n'a pas le droit de se préoccuper de la situation particulière d'énonciation que si le texte est ambigu. Lorsque le texte est clair, ils se fondent sur le sens littéral, même en cas de contradiction flagrante avec ce que le testateur semble avoir voulu dire. 
3 L'interprétation des testaments soulève d'autres problèmes. Il est légitime de supposer que le testateur en tant qu'individu avait bien une intention précise, mais il n'est plus en mesure de la préciser. Des problèmes d'interprétation se posent donc fréquemment, $\mathrm{y}$ compris pour déterminer la référence aux individus. Si les noms propres et les descriptions définies ont en commun la fonction de dénoter les individus, ils ne fonctionnent pourtant pas de la même façon.

\section{Noms propres}

Dans le modèle associé à J. S. Mill (1843), les noms propres n'ont pas de sens en soi. Ils ne servent qu'à désigner l'individu dont il est question sans rien en dire, un peu comme s'il s'agissait «d'une marque sur la porte ». Même si ce modèle semble bien correspondre dans la pratique aux besoins des juges, les objections théoriques sont bien connues.

Si la fonction des noms était effectivement de désigner sans décrire, alors un nom qui ne correspondrait pas à un référent dans le monde réel n'aurait aucun sens. La fiction deviendrait alors impossible. D'autre part, une déclaration de non-existence serait forcément contradictoire. En effet, une phrase comme "Harry Potter n'existe pas » n'aurait de sens que si elle était fausse. Enfin, si deux noms désignent le même référent, des affirmations d'identité comme 'Mohammed Ali est Cassius Clay' ou 'Superman est Clark Kent', ne seraient plus que des tautologies. Cela reviendrait à dire que 'Clark Kent est Clark Kent', sans apporter de nouvelles informations.

5 Afin de résoudre ce problème, Frege (1892) propose de distinguer le 'sens' de la 'dénotation' (Sinn et Bedeutung). Ainsi, puisque les expressions «l'étoile du soir» et « l'étoile du matin » correspondant toutes les deux à la même planète, la dénotation est identique. Mais il existe néanmoins une différence de sens. Malheureusement, Frege présente ainsi le 'sens' comme une entité privée, plutôt que comme une propriété publique, susceptible d'être saisie par les sujets-parlants différents.

6 Selon d'autres philosophes (Russell, 1905), le nom propre aurait au contraire la fonction de décrire l'individu désigné. Il s'y attacherait précisément parce qu'il équivaudrait à l'ensemble de descriptions vraies concernant cette personne. Ce modèle se heurte à des objections encore plus fortes. D'une part, étant donné que les sujets-parlants ne sont pas omniscients, ils ne peuvent jamais connaitre l'ensemble des descriptions vraies, et ne peuvent donc pas connaitre le vrai sens des mots qu'ils utilisent. Et de l'autre, puisque les différents interlocuteurs ne comprennent pas forcément le nom de la même façon, cela empêcherait la communication. Enfin, en cas d'erreur concernant la validité de certaines descriptions, le nom ne correspondrait plus à l'individu concerné. Ce problème est présenté par Wittgenstein (1953: 79) dans son paragraphe sur le nom de 'Moïse'. Selon Wittgenstein, si on dit que Moïse n'a jamais existé, cela pourrait signifier plusieurs choses, par exemple que les Israélites n'avaient pas de chef unique quand ils se sont retirés de l'Égypte, ou que leur chef ne s'appelait pas Moïse, ou que personne n'a accompli tout ce qui est relaté dans la Bible à propos de Moïse.

7 Wittgenstein poursuit : « Le nom 'Moïse' pourrait être défini selon plusieurs descriptions, par exemple, comme 'celui qui a mené les Israélites à travers le désert', ou 'celui qui a vécu à cette époque et à cet endroit et qui s'appelait 'Moïse'. »" Il en conclut que " selon que nous prenons une définition ou une autre, l'affirmation 'Moïse n'a pas existé' acquiert un sens différent, comme pour toute autre affirmation au sujet de Moïse ». 
8 Mais dans le monde de tous les jours, que ce soit en langue ordinaire ou en langage juridique, il est rarement nécessaire de donner une définition exhaustive du sens des noms propres. La plupart du temps les sujets-parlants arrivent à communiquer de manière convaincante même s'ils ne disposent que d'informations très partielles concernant la personne visée, dont certaines sont peut-être fausses. Il semble possible par exemple de parler de Jonas, le personnage Biblique censé avoir été avalé par une baleine, même si tout ce que l'on 'sait' sur lui est en fait faux. Ainsi, la théorie russellienne prévoit des difficultés pratiques qui ne se présentent pas dans la réalité.

Kripke (1972) propose une théorie plus satisfaisante de la référence, qui permet de rendre compte de manière plus réaliste de l'utilisation des expressions référentielles. Dans sa théorie causale, l'emploi des noms propres est considéré comme une pratique linguistique (au sens de Wittgenstein (1953)). Il suffit d'une sorte de 'baptême' à l'intérieur d'une communauté linguistique pour permettre l'emploi compréhensible d'un nom. Il n'est plus nécessaire de disposer d'une description complète de l'individu désigné. Au contraire, les noms sont associés à un certain nombre de croyances qui ne sont ni nécessaires ni analytiques. Puisque la communication ne dépend plus de faits réels, la référence reste possible même après l'abandon de certaines croyances. Cela permet alors d'échanger les idées au sujet de personnages fictifs, mais aussi, lorsque deux noms font référence au même individu, de rendre compte des affirmations d'identité.

Encore une fois, Wittgenstein (1953:79) rend compte du principe en prévoyant les problèmes que cela implique : il se demande si, lorsqu'il affirme quelque chose à propos de Moïse, il est toujours disposé à substituer l'une ou l'autre des descriptions possibles pour 'Moïse' :

J'entends peut-être par 'Moïse' celui qui a fait ce qui est relaté dans la Bible à propose de Moïse, ou en tout cas une bonne partie. Mais il n'est pas clair combien d'affirmations doivent s'avérer fausses avant que j'abandonne l'affirmation comme erronée. Est-ce que le nom 'Moïse' a 'un usage fixe et sans équivoque dans tous les cas possibles'? (Wittgenstein, $1953:$ : 79).

11 La théorie causale est corroborée en quelque sorte par l'interprétation des noms propres dans les testaments.

\subsection{Noms propres dans les testaments}

Les testateurs se trompent quelquefois sur le nom des héritiers. Nous trouvons un exemple dans l'affaire NSPCC $v$ SNSPCC 1915.

Dans cette affaire, un Écossais, Alexander Nasmyth, légua $£ 500$ à la 'National Society for the Prevention of Cruelty to Children' (la NSPCC). Néanmoins, puisqu'il paraissait clair que le testateur avait l'intention de faire bénéficier la société écossaise du même nom (la 'Scottish National Society for the Prevention of Cruelty to Children', SNSPCC), la Court of Session (en Écosse) désigna la SNSPCC comme héritière, mais cette décision fut inversée en appel par la Chambre des Lords (à Londres). Les Lords avaient certes l'impression d'aller à l'encontre de la volonté du testateur; mais en l'absence de toute ambigüité textuelle, ils ont préféré éviter de créer un précédent qui pourrait avoir des conséquences néfastes.

13 Lord Loreburn niait l'existence d'une règle rigide selon laquelle, lorsqu'une personne est régulièrement nommée dans un testament, il doit nécessairement hériter. Pour lui, il s'agissait non pas d'une règle, mais d'une présomption. Selon le juge: «La vraie 
justification sur laquelle il faut fonder la décision dans cette affaire est ceci, que l'emploi exact d'un nom crée une présomption forte contre quiconque ne possède pas le nom mentionné dans le testament. "

Pour justifier la prise en compte du contexte, il aurait fallu d'abord constater une ambigüité, mais « dans cette affaire, je ne crois qu'aucune ambigüité n'a été établie » ( NSPCC $v$ SNSPCC HL 1915, per Lord Loreburn).

Dans une autre affaire, Re Smalley CA 1929, un testateur semble s'être trompé sur le nom de sa femme.

Marié en 1899, Smalley quitta sa femme Mary Ann en 1910. Vers 1915, il fit un mariage bigame avec Eliza Ann Mercer, veuve. Il vécut avec Eliza Ann jusqu'à sa mort, le nouveau couple étant considéré dans la communauté locale comme mari et femme. Son testament faisait état d'un don à "ma femme Eliza Ann Smalley ", alors que sa femme légitime s'appelait Mary Ann et sa maîtresse Mercer. Le juge Eves déclara que la description 'wife' (femme, épouse) permettait de résoudre l'ambigüité, et que sa femme légitime devait hériter. Mais cette décision fut inversée par la cour d'appel. Il semblait à Hanworth MR, que le testateur avait utilisé le mot 'wife' dans un sens "secondaire » et que Eliza Ann Mercer devait hériter, puisqu'elle était réputée être sa femme à la date du décès. À son avis : « Pour ceux qui étaient en contact avec le testateur pendant la dernière partie de sa vie, $\mathrm{M}^{\mathrm{me}}$ Mercer était dans le rôle de sa femme. » Il lui semblait « très peu probable que le testateur se serait trompé au sujet du nom de sa femme ».

Les descriptions définies soulèvent plus souvent que les noms propres des problèmes d'ambigüité concernant l'identité de la personne désignée.

\section{Descriptions définies}

Si la seule fonction des descriptions définies était de désigner un individu, elles donneraient lieu, comme les noms propres, à des tautologies. Ainsi, pour prendre un exemple de Russell (1905), «Scott est l'auteur de Waverley » signifierait seulement que "Scott est Scott ». De même, la phrase "Gordon Brown est l'actuel Premier Ministre » signifierait seulement que «G. Brown est G. Brown ». De la même façon, toute affirmation de non-existence serait contradictoire. En effet, selon la théorie de Russell, il ne devrait pas être possible d'affirmer que «Le Roi actuel de France n'existe pas ». Si cette phrase était vraie, elle ne voudrait rien dire. Elle n'aurait de sens que si elle était fausse.

Cependant, à la différence des noms propres, les descriptions définies ne font pas que dénoter. Par définition, elles proposent une description qui permet en principe l'identification de l'individu désigné. Cela implique pourtant que lorsque la description est fausse, la description ne correspond plus à personne. Des problèmes de ce type se posent de manière récurrente dans l'interprétation des testaments.

\subsection{Descriptions définies dans les testaments}

Dans l'affaire Wagstaff 1907 un testateur légua ses biens à sa femme, ou à sa veuve, désignée ainsi dans son testament : «To my dear wife, D. J. Wagstaff; if she shall so long continue my widow, for her own use and benefit. »

Il est apparu par la suite que la dame avec qui il pensait être marié depuis 1884 était en fait la femme d'un dénommé A.G. Jalland, toujours en vie. Puisque son 'mariage' avec 
Wagstaff n'était pas légitime, elle n'était pas sa femme, et ne pouvait donc pas devenir sa veuve. Selon la théorie philosophique proposée de Russell, puisque la description donnée était fausse, le testament ne voulait rien dire du tout. Le juge Kekewich considéra ce résultat insatisfaisant, et préféra rechercher ce qu'il appelait un « sens secondaire »:

Je dois m'en sortir d'une manière ou d'une autre. Je n'ai pas le droit de dire que [le testateur] ne voulait rien dire. S'il a bien utilisé le mot 'veuve' dans un sens secondaire, mon devoir est de trouver qu'elle est ce « sens secondaire».

Le juge Kekewich décida donc que le testateur avait bien l'intention de léguer ses biens à celle qu'il croyait être sa femme. Ce jugement fut confirmé en appel par Cozens-Hardy MR, qui considérait que le nom primait sur la description. Pour Cozens-Hardy MR, il n'y avait aucun doute concernant l'identité de la personne visée. En effet, le testateur voulait certainement dire « la dame avec qui il a fait une forme de mariage à l'Église St Georges, à Hanover Square ».

Rappelons que Strawson (1950) a proposé une solution partielle à ce problème à l'aide de la notion de "présupposition pragmatique». Dans ce modèle, les mots ne font pas référence du tout. Au contraire, ce sont les locuteurs qui peuvent éventuellement les utiliser pour faire référence. Cependant, cette explication va à l'encontre de la tradition juridique d'interprétation littérale, puisque le sens dépend alors de l'intention plutôt que des mots utilisés.

\subsection{L'ambigüité systématique des descriptions définies}

Dans certains contextes, les descriptions définies sont ambigües. Selon le principe d'équivalence introduit par Kant, il devrait être possible de substituer une expression pour une autre ayant le même sens, sans changer la valeur de vérité de la phrase. Il en va ainsi pour "Gordon Brown est écossais " par rapport à "l'actuel Premier Ministre de l'Angleterre est écossais». Mais ce n'est pas toujours le cas, notamment lorsqu'il s'agit d'attitudes propositionnelles comme des croyances. Les gens n'étant pas omniscients, il est tout à fait possible, pour ceux qui ne suivent pas l'actualité, de croire que « Gordon Brown est écossais » sans nécessairement accepter la vérité de la phrase équivalente : « Le Premier Ministre est écossais. » Pour prendre un autre exemple, Lois Lane est réputée savoir que Clark Kent est journaliste, mais elle ne sait pas que la même chose est vraie de Superman. Il s'agit de la distinction de re et de dicto, bien connue depuis le Moyen Âge.

Une autre forme d'ambiguïté est observée dans les contextes modaux. Selon la théorie causale, les noms propres font référence de manière rigide au même individu dans tous les mondes possibles. Par exemple, une phrase comme «Gordon Brown aurait pu être anglais » laisse entendre que l'individu G. Brown aurait pu naitre au sud de la frontière entre l'Angleterre et l'Écosse. Cependant, la description définie correspondante: «L'actuel Premier Ministre aurait pu être anglais » à au moins deux lectures. Dans une lecture, cette phrase dit quelque chose à propos de l'individu Gordon Brown, comme avec le nom propre. Mais dans l'autre, l'expression ne dénote pas forcément un individu particulier. Elle peut aussi être interprétée comme signifiant qu'un autre membre du Parti Travailliste, notamment un Anglais, aurait pu prendre le poste de Premier Ministre. Il en va de même pour «Le Premier ministre aurait pu être Conservateur ", qui signifie dans une lecture possible qu'un autre parti politique aurait pu gagner les dernières élections. 


\subsection{Interprétations référentielles et attributives}

Pour Russell, les phrases comportant des descriptions fausses n'ont pas de sens, puisqu'elles présupposent l'existence d'un référent inexistant. Donnellan (1966) montre qu'il s'agit là d'une simplification, et que les descriptions définies devraient plutôt être considérées comme systématiquement ambigües. Il prend l'exemple suivant: «Le meurtrier de Smith est fou. »

Il est possible qu'en utilisant la description définie, «le meurtrier de Smith », le locuteur entende désigner Jones, peut-être parce que c'est Jones qui a été mis en examen. Si ses interlocuteurs sont également au courant de cette mise en examen, la phrase pourra très bien être comprise dans ce sens, même si Jones n'a pas encore été reconnu coupable, et même si les autres personnes ne sont pas forcément d'accord sur son éventuelle culpabilité. Il s'agit alors de l'emploi référentiel.

Donnellan va plus loin : même si nous découvrons par la suite que Smith s'est suicidé, et qu'il n'y pas eu de meurtre, il reste possible que le locuteur veuille désigner Jones, même sous une description fausse. En revanche, si le locuteur ne connait pas l'identité du meurtrier, et ne soupçonne personne en particulier, alors, même en utilisant la même expression au même moment, il ne fait référence à aucun individu. Dans ce nouveau contexte, l'expression ne dénote plus Jones en particulier, mais le meurtrier qui qu'il soit. Il s'agit de l'emploi attributif.

Il ne semble pas possible de décider de manière catégorique si une description définie dans une phrase donnée doit être qualifiée de référentielle ou attributive. Donnellan fait remarquer, au contraire que «le fait d'utiliser une description définie de manière référentielle ou attributive est une fonction des intentions du locuteur dans un cas spécifique ». Il ne lui semble pas plausible de « rendre compte de ce problème en termes d'une ambigüité au niveau de la phrase (ni syntactique ni sémantique - peut-être pragmatique) ». Comme dans la théorie de présupposition pragmatique de Strawson, ce sont donc les sujets-parlants qui font référence à l'aide de l'expression linguistique.

Cela veut dire que, contrairement à la théorie supposée admise par les juges, l'interprétation purement littérale ne permet pas de saisir l'intention du locuteur. Pour appréhender cette intention, il faut au contraire une interprétation contextuelle au cas par cas. La distinction entre les interprétations référentielle et attributive est donc à l'origine de véritables problèmes dans l'interprétation des testaments.

\subsection{Interprétations référentielles et attributives dans les testaments}

La distinction entre interprétation référentielle et interprétation attributive est bien connue des juges au moins depuis le XIX ${ }^{e}$ siècle, même s'ils n'utilisent pas les mêmes termes pour en parler. Le problème apparait clairement dans l'affaire Re Whorwood 1887. Whorwood légua une coupe en argent au Lord Sherborne, mais celui-ci mourut avant le testateur, le fils aîné héritant alors du titre. À la date du décès de Whorwood, il y avait donc un nouveau Lord Sherborne. Le tribunal dut décider si celui-ci devait hériter.

Étant donné que tous les autres bénéficiaires étaient désignés par leur prénom et que le testament précisait en outre que la coupe en question devait rester ensuite dans la famille de l'héritier, il semble raisonnable de considérer que le testateur entendait l'expression «Lord Sherborne» de manière attributive. La cour refusa néanmoins cette 
interprétation, considérant qu'à la date du testament, l'identité de Lord Sherborne était claire, et que l'expression devait être interprétée de manière référentielle. Selon le juge North J :

À l'époque où le testament fut rédigé, il n'y avait aucun doute concernant l'identité du Lord Sherborne. Il n'y avait qu'une seule personne qui avait droit à cette appellation. Personne n'aurait pu hésiter pour dire qui était Lord Sherborne. Certes, le testament contenait des termes qui montrent que le testateur voulait que la coupe reste ensuite dans la famille de Lord Sherborne. Mais ce fait ne change pas l'interprétation du don, qui visait le Lord Sherborne de l'époque. (Re Whorwood 1887, per North J).

Cette décision fut confirmée en appel par Cotton LJ, qui considérait que la cour devait éviter autant que possible de tenir compte du contexte particulier. Pour lui :

La première question est de savoir si la cour peut admettre des preuves concernant l'intention du testateur. Je suis d'avis qu'elle ne peut pas le faire. Des preuves ont été admises avec raison pour montrer que l'ancien Lord Sherborne était mort lorsque le testateur a rajouté le dernier codicille, mais je ne crois pas que ceci ait quelque effet que ce soit sur l'interprétation de cette clause du testament. S'il avait été bien conseillé, il aurait rédigé son testament différemment. (Re Whorwood 1887, per Cotton $\mathrm{LJ})$.

Dans l'affaire Re Boddington 1882, le testateur avait de bonnes raisons à la date du testament de se croire marié. Pourtant la description «my wife» était juridiquement fausse. Boddington légua $£ 200$ à sa «femme " plus un paiement annuel "pendant son veuvage ». Malheureusement, par la suite, le mariage fut déclaré nul et non avenu pour cause d'impuissance. Par conséquent, le mariage devait être considéré comme inexistant, non seulement à partir de la déclaration de nullité, mais depuis toujours. Concernant le don de $£ 200$, la cour adopta néanmoins l'interprétation référentielle, considérant que la description définie, même fausse, permettait d'identifier la personne visée. Mais le paiement annuel fut refusé, la cour considérant que la description devait cette fois être interprétée de manière attributive. Pour le juge Fry :

Il me semble que le paiement annuel lui est donné pour une période qui ne pourra jamais exister. Elle n'a jamais été la veuve du testateur, et elle ne pourra donc jamais continuer comme sa veuve pendant aucune durée. Par principe, alors, je ne vois pas comment un paiement annuel pendant une période inexistante puisse être revendiqué. (Re Boddington ChD 1882, per Fry J).

Ce résultat fut confirmé en appel, Lord Cotton distinguant clairement entre le don et le paiement annuel :

Dans le cas du don qui lui est fait en tant que sa femme, il n'y a qu'une fausse démonstration; la description comme femme ne fait que désigner l'individu. Ce n'est pas le cas, et ne peut être le cas, pour ce qui concerne la référence au veuvage. La référence au veuvage n'est pas faite uniquement pour désigner la personne, mais pour indiquer et pour définir la durée, le début et la fin de ce paiement annuel. (Re Boddington HL1884, per Lord Cotton LJ).

Lord Selborne LC précise en outre qu'il n'est pas possible de se départir des mots utilisés :

Le paiement annuel est effectué avec des termes qui expriment une condition selon laquelle elle doit rester sa veuve, et le paiement annuel doit continuer uniquement tant qu'elle garde ce statut, et nous ne pouvons pas nous départir de ses mots. (Re Boddington HL1884, per Lord Selborne LC).

32 La cour déclara alors que selon la règle de droit, nul ne peut hériter s'il est désigné sous une caractéristique qui n'est pas remplie. 
Plus d'un demi-siècle plus tard, dans l'affaire Re Gale 1941, des faits semblables se sont présentés. Le testateur a voulu donner un paiement hebdomadaire de $£ 1.5 \mathrm{~s}$. à sa compagne, Dorothy Marie Laing, " pendant son veuvage pour une période de douze ans ", ainsi que l'usufruit de sa maison à Leeds "pendant son veuvage ». Mais officiellement, le couple ne s'était jamais marié. Farwell J se considérait alors lié par la règle précédente décidée dans Re Boddington. Même si elle vivait avec le testateur, elle n'était que sa compagne et elle ne pouvait jamais devenir sa veuve.

D’après les exemples qui viennent d'être donnés, il semble qu'il y ait une présomption juridique en faveur de l'interprétation référentielle, qui est adoptée systématiquement en cas de doute. Mais l'interprétation référentielle ne semble pas toujours adéquate. Dans Re Amyot 1904, le testateur légua une propriété du nom de Golden Grove à l'un de ses neveux, dans les termes suivants : " To the eldest son of my sister Frances McKean Gibney and his heirs for ever. "

Mais le fils ainé mourut avant le testateur. La question se posa alors de savoir si le second fils devait hériter, étant donné qu'à la date du décès, étant le seul survivant, il était logiquement devenu le plus âgé. Comme dans Re Whorwood, Lord MacNaghton considéra qu'il fallait éviter autant que possible de tenir compte du contexte. La Chambre des Lords adopta donc l'interprétation référentielle, les juges supposant que le testateur visait la personne qui répondait à la description à la date de rédaction du testament. Lord MacNaghton souligna que : «Il n'y a rien dans le contexte pour justifier un éloignement du sens propre et ordinaire des mots utilisés. » Le cadet ne toucha donc pas l'héritage.

Dans l'affaire Re Hickman 1948, une testatrice légua un collier à sa belle-fille, puis à la mort de celle-ci « To the wife of my grandson absolutely ». À la date du décès en 1914, le petitfils (Sir Alfred Edward Hickman, Baronet), n'était pas encore marié. Par conséquent, aucun individu ne remplissait la condition. En 1919, pourtant, Sir Alfred épousa Lilian Williams, dont il divorça en 1940, pour épouser en deuxièmes noces l'actuelle Dame Nancy Hickman. Ainsi, lorsque l'affaire est arrivée devant les juges, Harman J put la qualifier de « concours entre deux dames".

Harman J supposa que la testatrice avait voulu désigner l'une des deux comme héritière, même si au moment de rédiger son testament, elle ne connaissait ni l'une ni l'autre. Comme le juge ne s'autorisait pas le droit de spéculer au sujet du choix que la testatrice aurait finalement fait, il choisit de suivre la règle énoncée dans le manuel Jarman on Wills, selon laquelle la personne qui remplit la première la description doit hériter : «Je crois que la première personne qui répond à la description de femme du petit-fils est celle qui prend le bijou, et qu'il n'y a rien qui peut le lui enlever. » Le collier est donc allé à la première des femmes du petit-fils.

Il est rare de trouver un testament dont le texte renvoie explicitement à l'interprétation attributive, rendant ainsi impossible l'interprétation référentielle.

William Thompson rédigea son testament en urgence en 1942 peu avant de partir à la guerre comme sous-lieutenant de la marine (Royal Navy). Il n'était pas encore marié, mais, prévoyant, il voulait néanmoins léguer ses possessions à celle qu'il allait peut-être épouser un jour. Il légua donc tout ce qu'il possédait à sa «femme, qui qu'elle soit » : «I, William Gilmour Thomson [...] do hereby in the event of my being married bequeath everything that I possess to my wife whoever she may be. »

Il a pu se marier avant de partir à la guerre, mais il n'en est jamais revenu, étant mort en mer trois mois plus tard. Le tribunal n'eut pas de problème pour identifier sa veuve. 
Néanmoins, étant donné la complexité des dispositions supplémentaires, l'affaire est passée devant la justice à deux reprises, en 1946, et en 1969, sous le nom de Thomson's Trustees $v$ Thomson et de Thomson's Trustees $v$ Keddie.

\section{Conclusion}

Bien que les juges anglais affirment souvent se fonder sur l'interprétation littérale, ils sont bien obligés de tenir compte des éléments contextuels, y compris pour déterminer le sens des expressions référentielles dans les testaments. Ils essayent couramment d'éviter ce problème en évoquant la notion de "sens littéral en contexte ». D'après la pratique observée, cette expression, qui semble à première vue relever de l'oxymore, correspond en fait à l'idée contextualiste de "ce qui est dit» (what is said). Cette "signification sémantique » est établie dans un contexte général d'arrière-plan, et se distingue ainsi du "sens pragmatique", qui comprend les implicatures possibles dans un contexte particulier.

41 Les juges semblent donc admettre, comme les contextualistes modernes, que le sens des mots dépend non pas d'une définition fixe et objective, mais des connaissances communes à l'intérieur de la communauté linguistique à un moment donné. Dans l'affaire Doe $v$ Hiscocks 1839, Lord Abinger soutient effectivement que dans une telle communauté, les mots peuvent avoir des significations particulières, inaccessibles aux étrangers. Malgré son respect pour la règle littérale, Lord Abinger considère alors que pour comprendre un testament, il est nécessaire de tenir compte de toutes les circonstances. Pour lui :

Le testateur appelait peut-être habituellement certaines personnes ou certaines choses par des noms particuliers, inconnus des autres. Si ces noms apparaissent dans son testament, il n'est donc pas possible de les expliciter ou les interpréter sans l'aide de témoignages pour indiquer la signification particulière que le testateur leur donnait, comme si son testament était rédigé dans un code secret ou dans une langue étrangère. (Doe v Hiscocks HL 1839, per Lord Abinger).

Cette observation correspond bien au fait que les sujets-parlants parlent différemment dans des contextes différents, par exemple, dans des interactions sociales, professionnelles ou familiales. Dans l'interprétation des testaments, les juges peuvent donc attribuer à un mot, non pas le « sens littéral » strict, mais la signification qu'il prend habituellement dans la communauté pertinente, tout en rejetant néanmoins des interprétations purement pragmatiques qui pourraient apparaitre dans un contexte plus spécifique.

Dans le testament le plus court qui n'ait jamais été reconnu valable (Thorn $v$ Dickens 1906), le juge a retenu la signification attribuée habituellement au mot «mother» dans le contexte familial. Le testateur avait écrit, la veille de sa mort, les trois mots. «All for mother " (Tout pour maman). Pourtant sa mère était déjà morte. Le tribunal accepta quasiment sans discussion que le mot «mother ", pris dans son sens habituel, désignait en fait sa veuve.

L'analyse juridique des expressions référentielles dans les testaments montre ainsi que ces expressions peuvent se révéler ambigües, et que leur interprétation doit dépendre dans une certaine mesure du contexte. La règle littérale ne peut donc pas être prise au sens littéral. 
Au-delà des seules expressions référentielles, les juges de la common law sont amenés à traiter d'autres problèmes linguistiques, toujours en analysant des exemples authentiques. Même si, pour des raisons qui relèvent des contraintes professionnelles spécifiques, les solutions proposées ne correspondent pas toujours à l'analyse linguistique classique, leur raisonnement semble toujours clair, et cohérent. Étant donné que les juges soulèvent souvent de nouvelles questions sémantiques intéressantes, la linguistique juridique ne devrait donc pas être ignorée.

\section{BIBLIOGRAPHIE}

DONNELLAN K. (1966) : Reference and definite descriptions, Philosophical Review, 77, p. 281-304.

FREGE G. (1892) : Sinn und bedeutung, Zeitschriffte für Philosophie und philosophische Kritik, vol. 100, p. 25-50) (= On sense and meaning, dans P. Geach et M. Black (dir.) (1952) : Translations from the philosophical writings of Gottlob Frege, Oxford, Blackwell).

KRIPKE S. (1972): Naming and necessity, dans D. Davidson et G. Harman (dir.), Semantics of natural language, Dordrecht, Reidel.

MILL J. S. (1843) : System of Logic, dans J. M. Robson (dir.), Collected Works (1947) Toronto, University of Toronto Press.

RECANATI F. (2004) : Literal meaning, Cambridge, CUP.

RUSSELL B. (1905) : On denoting, Mind, 14/56, p.479-493.

SEARLE J.R. (1979) : Le sens littéral, Langue française, 42, p. 34-47.

STRAWSON P. (1950): On Referring, Mind, 59, dans Logico-linguistic papers (1971), London, Methuen, p.1-27.

WITTGENSTEIN L. (1953) : Philosophical Investigations, Oxford, Blackwell.

\section{Affaires citées}

Re Amyot (Amyot v Dwarris) PC [1904] AC 268.

Re Boddington [1883] 22 ChD. 597; HL [1884] 25 ChD. 685.

Boyes $v$ Cook [1880] 14 ChD 53.

Doe v Hiscocks HL (1839) 5 M. \& W. 363.

Re Gale [1941] ChD 209.

Re Hickman's Will Trusts [1948] ChD 624.

Ingram $v$ Little [1961] 1 QB 31.

Kingsbury $v$ Walter HL [1901] AC 187.

Lewis v Avery [1971] 1 QB 198.

Nasmyth $v$ NSPCC [1812] IC 2Div 16. 
NSPCC $v$ SNSPCC HL [1915] AC 207 (Law Times III 869-71).

Phillips $v$ Brooks Ltd [1919] 2 KB 243.

Re Smalley (Smalley $v$ Scotton) CA 192973 Sol Jo 234.

Thomson's Trustees $v$ Thomson [1946] IH 2 Div 41; [1955] IH 1Div 427.

Thomson's Trustees $v$ Keddie [1969] 1H 2Div 18.

Thorn $v$ Dickens, Probate (1906) WN 54.

In Re Wagstaff(Wagstaff v Jalland) ChD [1907] HL [1908] 1 Ch. 162.

In Re Whorwood (1887) 34 ChD 446.

\section{NOTES}

1. Cette citation et les suivantes ont été traduites par l'auteur.

\section{RÉSUMÉS}

Lorsque des problèmes se posent dans l'interprétation des testaments, les juges anglais sont tenus de suivre la 'règle littérale', et de retrouver l'intention du testateur uniquement par l'analyse des mots utilisés. Cependant, ils sont souvent amenés à tenir compte du contexte, y compris pour déterminer la référence des noms propres ou des descriptions définies. L'interprétation juridique des testaments authentiques fait ainsi apparaitre des problèmes sémantiques intéressants. Même si les juges doivent respecter des contraintes spécifiques, leur raisonnement semble cohérent et explicite, et ne doit pas être ignoré. Leurs conclusions semblent corroborer dans une certaine mesure la théorie sémantique contextualiste.

When problems arise in the interpretation of wills, English judges are required to follow the 'literal rule' and to discover the intention of the testator solely through the analysis of the words used. However, it is often necessary to take account of the context even to determine the intended reference of proper names or definite descriptions. The judicial interpretation of wills is seen to raise interesting semantic problems. Although the judges must respect specifically legal constraints, their reasoning seems coherent and explicit; and should not be ignored. Their conclusions seem to corroborate to a certain extent the theory of contextualist semantics.

\section{AUTEUR}

\section{ROSS CHARNOCK}

Université Paris-Dauphine 